\title{
Cortical Blindness and Ataxia Complicating Bronchial Artery Embolization for Severe Hemoptysis
}

\author{
Guoping Peng ${ }^{1}$, Hui Liang ${ }^{1}$, Lingxiang Ruan ${ }^{2}$ and Benyan Luo ${ }^{1}$
}

\begin{abstract}
Complications of bronchial artery embolization (BAE) are uncommon. A 37-year-old patient with pulmonary tuberculosis received bronchial artery embolization because of severe hemoptysis. The bilateral bronchial arteries and left internal mammary artery were embolized using a gelatin sponge, and the patient exhibited occipital blindness and ataxia after the second BAE. The dissolvable gelatin sponge possibly entered the posterior circulation, resulting in the multiple infarctions in the bilateral occipital lobes and cerebellum. Because of the bad prognosis and the difficulty for curability, this kind of complication should be recognized in a timely manner and carefully avoided by the interventional radiologists carrying out the BAE.
\end{abstract}

Key words: bronchial artery embolization, cerebellar infarction, cortical blindness, hemoptysis

(Inter Med 49: 1445-1447, 2010)

(DOI: 10.2169/internalmedicine.49.3244)

\section{Introduction}

Bronchial artery embolization (BAE) is commonly used in patients with massive hemoptysis, however, it should be cautiously carried out to prevent vascular complications associated with the procedure (1). We report herein a case of multiple infarctions in the posterior circulation which were exhibited as occipital blindness and ataxia complicating BAE.

\section{Case Report}

A 37-year-old man with pulmonary tuberculosis identified by CT scan (Fig. 1A) was admitted to the lung unit for severe hemoptysis (about $700 \mathrm{~mL}$ in 24 hours). The patient was a smoker and his medical history was unremarkable. In addition, the patient showed no signs of a fever and was hemodynamically stable. Fiberoptic bronchoscopy showed an active bleeding in the apical segment of the left upper lobe, and the selective bronchial artery angiography was then performed and there was no aortic atheroma. The bronchial angiography indicated a tortuous left bronchial artery as the source of bleeding (Fig. 1B); the right bronchial ar- tery was also enlarged though no sign of bleeding was seen, thus, the bilateral bronchial arteries were both embolized using a gelatin sponge. The left bronchial artery and internal mammary artery were embolized for a second time as the patient still had episodes of hemoptysis after the first BAE. An aortic backflow of the contrast media was found during the control angiogram. After the second BAE, the bleeding of the affected artery was successfully stopped (Fig. 1C), and the hemoptysis never recurred. The patient seemed to be sleepy and not very alert when he was shifted to the ward. He was then given an emergent cranial CT but no intracranial lesion was found. After six hours of lying in bed, the patient complained of a slight headache and dizziness when he was trying to get up. He also vomited several times and complained of unclear vision when he was trying to walk. Neurologic examination showed that the light reflex and the extra-ocular movements were normal but there was a bilateral loss of vision. His right arm was slower than the left for rapidly alternating movements. Finger to nose testing showed postpointing of right hand. His Romberg testing was positive. His mental status was normal. The ophthalmoscopy showed no evidence of central retinal artery occlusion. A second non-contrast cranial CT showed multiple hypodensities in the cerebellum, and the follow-up cranial MRI scan

${ }^{1}$ Neurology Department, First Affiliated Hospital, Medical College, Zhejiang University, China and ${ }^{2}$ Radiology Department, First Affiliated Hospital, Medical College, Zhejiang University, China

Received for publication December 14, 2009; Accepted for publication March 25, 2010

Correspondence to Dr. Benyan Luo, luobenyan@zju.edu.cn 

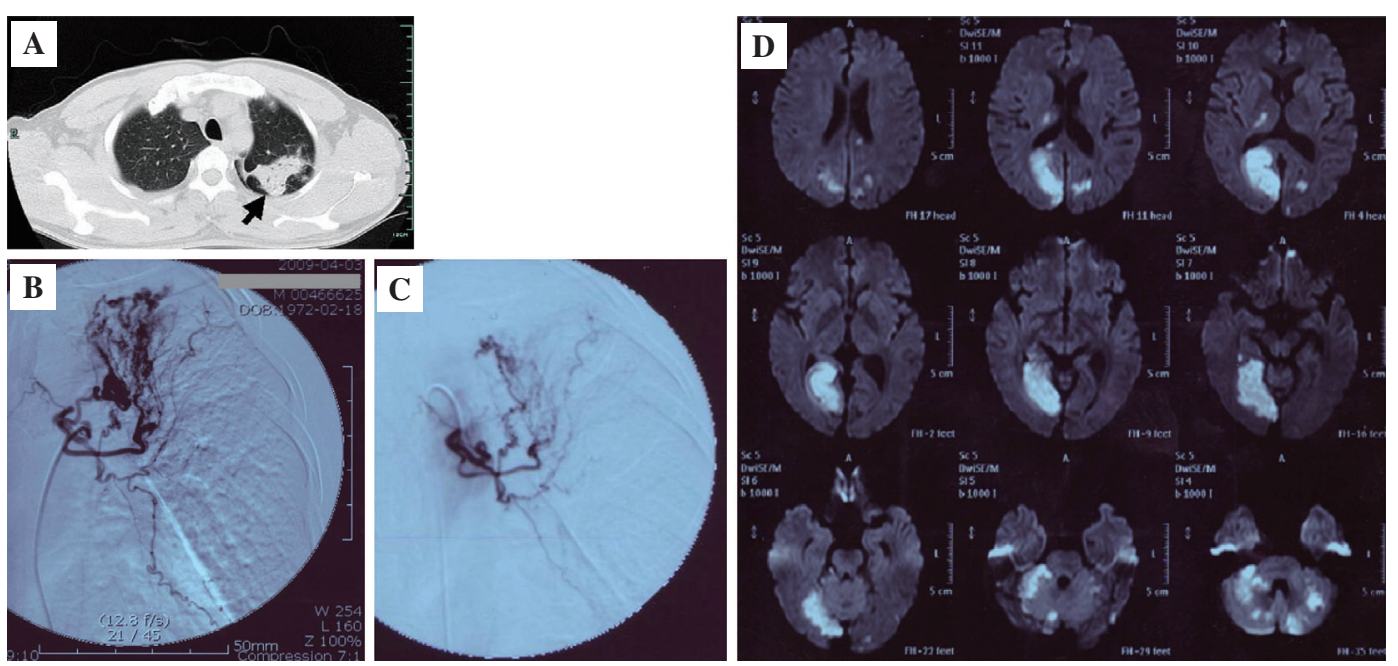

Figure 1. (A) Pulmonary CT shows an infected lesion located in the upper left lobe. (B) Left bronchial arteriogram demonstrates a tortuous, enlarged bronchial artery and its branches supplying a hypervascular lesion in the left upper lobe. (C) Selective postembolization angiogram demonstrates no hypervascular lesion in the left upper lobe. (D) Diffusion-weighted images show multiple areas of different sizes of focal infarcts in a posterior circulation distribution. Imaging was performed 2 days after the bronchial artery embolization.

showed multiple infarctions within the cerebellum and both sides of the occipital lobe (Fig. 1D). There was no significant finding in the cranial MRA, the carotid ultrasonogram or the echocardiogram. The patient received conservative management for ischemic infarction using antiplatelet drugs and other therapy for improving cerebral microcirculation. Thirty days later, the patient was discharged from hospital with normal coordination and almost normal visual fields. The further course was uneventful at the 6-month follow-up.

\section{Discussion}

The efficacy of BAE as the first-line treatment of severe hemoptysis has been undoubtedly demonstrated in the past decade, with an immediate control of bleeding in up to $90 \%$ of cases. However, serious vascular complications may occur in $<10 \%$ cases. The most disastrous complication of BAE is spinal cord ischemia due to the inadvertent occlusion of spinal arteries. Other complications following BAE have been reported in the literature including chest pain, bronchoesophageal fistula, fatal ischemic colitis, and also renal and splenic infarctions (2).

Previously, infarction with only posterior circulation distribution due to cardiac or idiopathic source of embolism was reported (3). As for the complication with BAE, two cases of transient cortical blindness following BAE were addressed by Liu et al (4). Recently, a case was reported for exhibiting only a minimal symptom of headache because of posterior circulation infarct after BAE (5). Here, we report a case of multiple cerebellar and occipital infarctions complicating BAE for severe hemoptysis. Since the cranial MRA clearly showed no stenosis and the carotid ultrasonogram showed no atherosclerotic plaques, and the patient had no history of hypertension or hyperlipidemia, the chance of thrombosis or cholesterol emboli secondary to the arterial catheterization is likely very little. Meanwhile, no evidence of an intra-cardiac source of embolism was found since the echocardiogram showed no atrial thrombus or neoplasms, or patent foramen ovale (6).

On the other hand, absorbable gelatin sponge is typically widely used for BAE because it is inexpensive, easy to handle, and has a controllable embolic size. However, disadvantages of its dissolvability and lack of radiopacity may lead to recanalization of the embolized artery and may sometimes be responsible for recurrent bleeding. Thus, the present patient received two times of BAE. As reported before, the embosphere particles during BAE may pass through the bronchiopulmonary shunt or through the collateral vessels into the systemic circulation, resulting in multifocal infarction $(4,7)$. In this case, the postulated mechanism is a reflux of the particles within the aortic lumen. Therefore, dissolvable gelatin sponge possibly entered the blood flow of the vertebral-basilar system and into the posterior distribution where cortical blindness and ataxia resulted as a consequence. However, the possibility of embolic particles entering the posterior circulation through collaterals between the bronchial and vertebral arteries could not be completely excluded yet.

Because of the severity and less favorable prognosis for the infarction in the posterior circulation, especially for ischemic lesions located in the brainstem, this kind of complication should be carefully avoided by the interventional radiologists carrying out the BAE. Special care should be taken to prevent reflux by slowly and equably injecting the embospheres under continuous fluoroscopic control if possible.

The authors have no conflicts of interest or financial support to 
disclose.

\section{References}

1. Fartoukh M, Khalil A, Louis L, et al. An integrated approach to diagnosis and management of severe haemoptysis in patients admitted to the intensive care unit: a case series from a referral centre. Respir Res 8: 11-20, 2007.

2. Ma KF, Wong WH, Lui CY, Cheng LF. Renal and splenic microinfarctions following bronchial artery embolization with tris-acryl microspheres. Korean J Radiol 10: 97-99, 2009.

3. Yamamoto Y, Georgiadis AL, Chang H-M, Caplan LR. Posterior cerebral artery territory infarcts in the New England Medical Center Posterior Circulation Registry. Arch Neurol 6: 824-832, 1999.

4. Liu SF, Li TY, Wong SL, Lin AS. Transient cortical blindness: a complication of bronchial artery embolism. Respir Med 92: 983-
986, 1998.

5. FitzGerald DB, Suran EL, Sargent J. Posterior circulation infarct after bronchial artery embolization and coiling. Neurology 65: 1312, 2005.

6. Hayashida K, Fukuchi K, Inubushi M, Fukushima K, Imakita S, Kimura K. Embolic distribution through patent foramen ovale demonstrated by ${ }^{99 m}$ Tc-MAA brain SPECT after valsalva radionuclide venography. J Nucl Med 42: 859-863, 2001.

7. Sriram KB, Taylor DJ, Holmes M. Systemic multifocal infarction following bronchial artery embolization with microsphere particles. Intern Med J 37: 734-735, 2007.

(C) 2010 The Japanese Society of Internal Medicine http://www.naika.or.jp/imindex.html 